\title{
A Distributed Database on the INTERNET of 3D Models of Human Pathological Organs
}

\author{
M. Crudele*, G. J. Clapworthy**, M. A. Krokos**, \\ G. Salcito***, N. Vasilonikolidakis**** \\ *Libero Istituto Universitario Campus Bio-Medico di Roma, \\ Via Longoni 83 - 00155 Rome, Italy \\ (crudele@liucbm.it) \\ ${ }^{* *}$ Department of Computer and Information Sciences, De Montfort University, \\ Hammerwood Gate, Kents Hill, Milton Keynes MK7 6HP, United Kingdom \\ (gc@dmu.ac.uk) \\ ***CITEC S.p.A., Via Alessandro Farnese 3 - 00192 Rome, Italy \\ (gsalcito@corelli.nexus.it) \\ ****Department of Research and Development, Neuroware Ltd., \\ 12 Alitsis Street, Athens 10433, Greece \\ (nikvas@neuro.ath.forthnet.gr)
}

\begin{abstract}
We describe a research project for the development of a system for cataloguing, retrieval and interactive manipulation of $3 D$ models of pathological human organs from a distributed WWW database. The project aims to provide transmission and rendering of three-dimensional models of human pathologies (constructed mainly from MRI and CT slices), enhanced manipulation and fly-through functionality on the models, as well as $3 D$ animation sequences of virtual endoscopy. The system supports standard $P C$ platforms without specialised hardware, thereby allowing accessibility to the widest possible audience. The system will provide the end users with advanced imaging capabilities such as models derived from multimodal inputs (CT and MRI), stereoscopic display and will incorporate a number of features such as model warping. In addition to interactive utilisation, the service will provide interactive platforms for education, incorporating monitoring and assessment, as well as for information and/or counselling exchange between users.
\end{abstract}

\section{Introduction}

There is an increasing interest in the medical academic world about the availability of three dimensional images of human organs. Most of the currently accessible databases are built on a the Visible Human project [1] material, which contains the entire cadaver of a male and a female, without specific pathologies; in some other cases you can find few images of pathological organs. The outcome of the IAEVA research European project (Telematics Applications for Healthcare: $\mathrm{HC1025}$ ), which will conclude its cycle at the end of the year, gives the chance of widening the initial aims for implementing an improved transmission and rendering of three-dimensional models of human pathologies (constructed mainly from MRI and CT slices), enhanced manipulation and fly-through functionality on the models, as well as 3D animation sequences of virtual endoscopy. A great emphasis is on the educational aspect, through the realization of a mentoring system and bulletin boards, together with self-evaluation tools [3]. We briefly describe this new project, which will involve technical and academic partners. 


\section{Background}

The methodology of the system developed so far followed these lines.

\subsection{Collection of user requirements}

We interviewed physicians of different specializations, as well as medical students, about the data structures, search procedures and visualization and interactivity modalities. The most common requirements were:

- Low cost hardware and software.

- Fast response times in downloading.

- Slicing of 3D images.

- Comparison of 3D volumetric images of human organs in the normal and pathological states.

\subsection{Survey of the WWW}

A survey of the world-wide availability of 3D visualisation tools and databases of humanorgan images on the INTERNET was conducted. By combining different expensive systems we would have been able to reach the main goal of reconstruction and interactive manipulation of $3 \mathrm{D}$ volumetric models. On the other end we could not find any low-cost and widely diffused system.

\subsection{Construction of a relational database of $3 \mathrm{D}$ volumetric models}

Our distributed relational database of $3 \mathrm{D}$ volumetric models of human organs, contains entities related to a human organ and entities related to parameterized tours of the database. We employ ODBC to communicate with the SQL based Relational database management system (RDBMS) and assume no particular vendors of ODBC or Database. The information linked to every model contains the SNOMED (Systematized Nomenclature of Human and Veterinary Medicine) [4] codes about topology (location in the body) and diagnosis.

\subsection{Construction of the Solid Model Producer}

The Solid Model Producer design is based on building our own $\mathrm{C}++$ classes on top of the freeware libraries of the Visualisation Toolkit (VTK) [9], which is platform independent and employs OpenGL. Any image processing enhancement (if necessary) can be done prior to 3D model production. Generally, we assume that external tools are employed to pre-process the data set (as captured by a CT, MRI etc. scanner) and that the SMP accepts as input a set of 2D slices and produces a 3D model.

\subsection{Construction of the Solid Object Viewer}

The Solid Object Viewer (SOV) is an independent software component that can read a 3D volumetric model (from a file) and subsequently render it. The user requests to view a 3D volumetric model, so the WWW server receives a request and the WWW server employs the Common Gateway Interface (CGI) to inquire the distributed database and thus retrieve the URL of the 3D model filename; this action, in fact, initialises the operation of the SOV. Now, 
the SOV reads the file, processes the data, prepares it, and sends it rendered to the computer screen and thus the user can send a number of requests for various operations for interactive manipulation (rotation around an arbitrary axis, zooming in/out, extraction of orthogonal planes corresponding to axial, sagittal and coronal cross-sections). The SOV performs the socalled incremental rendering i.e. the user rapidly gets a crude model on the computer screen which is then gradually refined as the server transmits more detail.

\subsection{Construction of the Database Connectivity Module}

The Database Connectivity Module (DCM) CGI program that is employed to interface the RDBMS to WWW servers is the heart of our system; its main function is:

- to present data entry requests to the user,

- to subsequently translate them into SQL format,

- to pass them to the RDBMS and finally

- to move the results back and present them to the user.

\subsection{Implementation of the resource manager for our database}

The DCM connects the WWW server software with the database engine i.e. the query mechanisms of the browser are served by the DCM. The implementation has being carried out under embedded $\mathrm{C} / \mathrm{C}++$ for cross platform portability under Windows NT with support of ODBC level 3. One important issue to handle at this stage is the identification of data servers. For our purposes, servers are scattered all over the INTERNET. Since the browser does not know how the database is physically scattered, it is the responsibility of the WWW server to handle this. We have also simplified the task of adding pages to the server by designing a small number of pages (say 10 to 15 for our WWW server) which we call prototypes, and subsequently allowing the WWW server to fill data in a prototype by sending a question (query) to a relational database that will be already filled in with the information needed by the pages of the WWW server.

\section{Project outline}

The primary goal for the service developed under this project is education. Setting up a system aimed to the clinical care would be very different, but we also consider that the first step to give physicians the capability of extending their knowledge is providing them with tools that do not imply any risk. Moreover, also physicians need to study, to refresh what they learned or to keep updated.

We are therefore moving further in the implementation of a distributed database of pathological organs on the WWW. The main new features are briefly described.

\subsection{Additional patient data}

The use of SNOMED allows us to extend the classification of the 3D models, by adding more data regarding the organ (morphology, function, procedures) and the patient (activity, occupation, social context). The use of SNOMED DICOM MICROGLOSSARY (SDM) [5], a resource for mapping DICOM coded entry Data Elements to the appropriate sets of terms from SNOMED International, is to be considered related to the future use of ACR-NEMA DICOM 3 standard applied to 3D images. 


\subsection{Bulletin board}

Each image or set of images can be linked to a specific bulletin board where commentaries to its characteristics are added by anyone accessing the database. These boards can be reserved to one group (e.g. a University Department) or open to everyone.

\subsection{Tutoring and mentoring system}

While the bulletin board is not moderated, and the quality or correctness of the information is not guaranteed, a more structured tutoring and mentoring system helps the student who accesses the database. It also means that a group of students can be followed by one or more teachers to have them reach the appropriate level of knowledge in that particular field.

\subsection{Case studies}

A collection of $3 \mathrm{D}$ models can form a case study, with all the annexed information. The case study can be periodically delivered to a group of registered students, allowing the tutor to monitor their responses.

\subsection{Certification of diagnosis and copyright issues}

All the data regarding diagnosis carry the physician's name and its workplace, so that he/she can eventually be contacted for clarifications. Obviously, no information that can lead to the identification of the patient will be recorded. Provided that the 3D models are distributed among the clinical and academic partners of the consortium, on different WWW servers, it is likely that every owner will keep all the rights on the original 2D images. A watermark system for 3D models will be studied and eventually applied for copyright [7].

\subsection{Multilingual support}

The system is entirely developed in English. The availability of SNOMED in other languages (e.g. French) can provide an automatic translation of the database information. The textual information can be automatically translated by means of modern translating software: the resulting quality would not be sufficient for publishing, but may prove to be enough for the comprehension of the content.

\subsection{Satellite delivery}

The need for very fast transmission can be fulfilled by the use of satellite Internet connections. The actual cost for the receiving equipment is affordable. The transmission fees are not very cheap, but they will surely be lower in the future. Moreover, if a broadcasting approach is used (for example for the delivery of case studies), the cost can be shared among all the users. TV satellites are likely to be the cheapest ones.

\subsection{Support of multimodal images}

This support allows images of the same organ originating from CT and MRI to be combined into one model. Such a model contains more structural information and provides better vifial 
cues to the end user. The current project focuses on combining images from CT and MRI, although other possibilities exist (hagiography, pyelotomy, etc).

\subsection{Model warping}

It is the process of transforming one solid to another one using space operators. This is an important feature which allows transformation of a normal organ to one exhibiting a pathology, to show the progressive modification of the structures.

\subsection{Production of 3D endoscopy animation sequences}

The Solid Model Producer can be enhanced to reconstruct virtual endoscopy from MRI slices. This will involve processing the MRI slices, setting proper viewing parameters per case and producing the model in one of the leading formats for interactive movies.

\subsection{Stereoscopic display}

In some cases the ability to produce stereoscopic display by use of shutter-glasses is very useful for teaching purposes. In fact, for a student it is not always very easy to understand the volumetric properties of a pathological organ, even though it is represented in a $3 \mathrm{D}$ projection. The distances and the relationships among the different structures are much clearer when using a real stereoscopic vision.

\section{Conclusions}

Currently, our system is under test and the new features described are being implemented in different phases. One issues is to widen the network of possible content providers (University Clinics or Hospitals) and to study the marketing strategy. The feasibility of this project is well beyond the volunteer scheme of an academic cooperation. If we want to get a full coverage of organs and pathologies there is a need for a permanent structure. That's why we have involved in the project major telecommunication companies, Internet publishers, medical imaging system manufacturers, as well as Universities and Hospitals.

\section{References}

[1] Ackerman-MJ; Spitzer-VM; Scherzinger-AL; Whitlock-DG. The Visible Human data set: an image resource for anatomical visualization. Medinfo. 1995; 8 Pt 2: 1195-8

(2) Hohne K.H, Pflesser B, Pommert A, Riemer M, Schiemann T, Schubert R. Tiede U. A 'virtual body' Model for Surgical education and rehearshal. IEEE Computer Magazine Jan 96, pp25-31.

[3] McEnery-KW; Roth-SM; Kelley-LK; Hirsch-KR; Menton-DN; Kelly-EA.. A method for interactive medical instruction utilizing the World Wide Web. Proc-Annu-Symp-Comput-Appl-Med-Care. 1995: 502-7

[4] http://www.snomed.org

[5] http://nelle.mc.duke.edu/standards/HL7/sigs/image-management/HTML/snomed-home.html

[6] Caramella-D; Neri-E; Del-Sarto-M; Lencioni-R; Bartolozzi-C. [Implementation of a server World Wide Web of radiology accessible by Internet] Realizzazione di un "server World Wide Web" di radiologia accessibile da Internet. Radiol-Med-Torino. 1996 May; 91(5): 622-6

[7] Stern-EJ; Westenberg-L. Copyright law and academic radiology: rights of authors and copyright owners and reproduction of information. AJR-Am-J-Roentgenol. 1995 May; 164(5): 1083-8

[8] Chen-LS; Chen-JP; Chen-SC; Liu-PW; Shyu-SS. A distributed and interactive three-dimensional medical image system. Comput-Med-Imaging-Graph. 1994 Sep-Oct; 18(5): 325-37

[9] Schroeder, W. Martin, K. Lorensen, B. : The Visualization Toolkit: An Object-Oriented Approach To 3D Graphics, Frentice Hall, 1996. 